\title{
Three-dimensional virtual reconstruction with DocDo: A novel interactive tool to score renal mass complexity
}

Bernardo Rocco ${ }^{1}$, Maria Chiara Sighinolfi ${ }^{1}$, Aurus Dourado Menezes ${ }^{2}$, Ahmed Eissa ${ }^{1,3}$, Raffaele Inzillo ${ }^{1}$, Marco Sandri ${ }^{4}$, Stefano Puliatti ${ }^{1}$, Filippo Turri ${ }^{1}$, Silvia Ciarlariello ${ }^{1}$, Marco Amato ${ }^{1}$, Cosimo De Carne ${ }^{1}$, Luca Sarchi ${ }^{1}$, Giorgio Bozzini ${ }^{5}$, Bianchi ${ }^{1}{ }^{1}$, Salvatore Micali ${ }^{1}$

1 Urology Department, University of Modena \& Reggio Emilia, Modena, Italy

2 Clinica da Urologia e Cirurgia Urologica, Teresina, Brazil

3 Urology Department, Faculty of Medicine, Tanta University, Tanta, Egypt

4 Data Methods and Systems Statistical Laboratory, University of Brescia, Brescia, Italy

5 ASST, Valle Olona, Italy

\section{Corresponding Author:}

Maria Chiara Sighinolfi, $\mathrm{PhD}, \mathrm{MD}$

University of Modena and Reggio Emilia, Italy

Phone: +393921329216

Email: sighinolfic@yahoo.com

This article has been accepted for publication and undergone full peer review but has not been through the copyediting, typesetting, pagination and proofreading process, which may lead to differences between this version and the Version of Record. Please cite this article as doi: $\underline{10.1111 / \text { bju. } 15049}$

This article is protected by copyright. All rights reserved 
Dear Editor,

The pre-operative knowledge of the complexity of a renal mass is the cornerstone to plan a partial nephrectomy(PN) [1]. Given the multiple issues characterizing it - size, protrusion out from renal parenchyma, longitudinal and coronal location, proximity to the hilum nephrometric scoring systems have been proposed during the last decade[2, 3]; RENAL and PADUA classifications are the mostly used [4-6]. However, such systems are based on conventional 2D-imaging rendered as a greyscale, thus implying a sort of cognitive 3Dreconstruction of a complex structure like the kidney. Porpiglia[1] addresses this unmet concern by scoring PADUA and RENAL systems with 3D-virtual models developed by MEDICS. As expected, 3D-nephrometric scoring performed better than the conventional 2Dbased in predicting post-operative complications.

Similarly, DocDo is a novel interactive online tool that provides a customized 3D virtual reconstruction of the kidney. As for the MEDICS one, it is developed from the collaboration of bioengineers and radiologists. Conventional DICOM images (four-phase CT-scan or MRI, at least 2,5-mm thickness) are sent to the DocDo platform which develops and shares within few days 3D-reconstruction of the kidney that could be displayed into an application available for iOS or Android. All anatomical structures are three-dimensionally depicted; the kidney is transparent - to enable the visualization of the inner structures and their relationship - and all elements could be discretionally removed to focus on the area of interest. The image could be moved and rotated and details could be enlarged by tapping on the screen (Figure1A).

We are currently using DocDo in the setting of robotic PN. Herein, we aimed to assess the DocDo effectiveness for the calculation of nephrometric scores and to evaluate variables affecting ischemia time (IT) and operative time (OT).

The database of our institute was reviewed to include all the consecutive patients with single renal mass, who underwent DocDo-planned robotic PNs. Between January and June 2019, a total of 10 patients were retrieved from the hospital records (eight patients diagnosed with CT and two with MRI scans). Figure 1B shows a summary of the patient's characteristics and the $3 \mathrm{D}$ and $2 \mathrm{D}$ nephrometric scores. Two patients underwent a concomitant robotic intestinal resection (for bowel tumor); a single patient underwent concomitant robotic cholecystectomy. All procedures were performed with the daVinci $\mathrm{Si}$, without conversion to radical nephrectomy and without selective clamping. Mean ischemia time was $17 \pm 9.1$ minutes. Mean OT was $144 \pm 36$ minutes (for patients with the concomitant procedure the OT was calculated as console time of the urological part). The median length-of-stay was $6 \pm 2.4$

This article is protected by copyright. All rights reserved 
days. No major complications were reported. Three patients had a Clavien-II (pleural effusion requiring antibiotics); none of patients needed blood transfusion.

Histopathology revealed a clear cell carcinoma in six patients, angiomyolipoma in two, oncocytoma in one, and an epithelial neoplasm in the last patient. All surgical margins were negative.

From a regression analysis, where IT and OT are considered as continuous variables, 3DPADUA score original grouping (coefficient $=9.25 ; 95 \%$ CI $1.97-16.53 ; \mathrm{p}=0.020$ ), CS proximity (coefficient $=20.25 ; 95 \%$ CI $5.46-35.04 ; \mathrm{p}=0.014$ ), radius for both PADUA and RENAL (coefficient $=11.7$; 95\% CI $1.25-22.15$; $\mathrm{p}=0.033$ ), and a hilar tumor (coefficient= $18 ; 95 \%$ CI $10.69-25.31 ; \mathrm{p}=0.001$ ), were the variables significantly related to IT. The radius for both PADUA and RENAL scores (coefficient=60; 95\% CI 24.08 - 95.92; p=0.006) was the only variable statistically related to operative time.

The current report is preliminary and aims to depict the feasibility of a 3D-scoring with a system other than the MEDICS one. From a subjective surgeon's perspective, this platform with its easy interface allowed to better navigate the renal anatomy, facilitate the dissection and the identification of the renal vessels.

However, the study presents some limitations: 1) small sample size; 2) the absence of Clavien-III complication avoided a regression analysis of related factors; 3) comparison with the prediction from 2D-PADUA and 2D-RENAL systems was not performed; and 4) the Length-of-stay may be affected in some patients by the presence of concomitant surgical procedure.

The DocDo applications does not allow measurement of distances as it is not provided with a ruler. This occurrence makes the evaluation of some items (as nearness to CS) assessed in a cognitive way referring to other known distances (ie radium as a reference-marker).

Our analysis confirmed the findings of Porpiglia [1], that the lowest agreement between the 2D- and 3D scores was found in the variables related to the protrusion of the mass and its relationship with the hilum (Figure 1B). Thus, the 3D-scoring may potentially provide better advantage in the assessment of these features; a prospective comparative study is currently ongoing, as well as technological improvements like the inclusion of a ruler inside the tool.

As stated by Porpiglia [1], a novel era is ready to start with the 3D-imaging reconstruction: in a scenario with growing evidences favoring a conservative approach, trying to expand indications to PN and to elect more and more tricky cases, 3D-models may overcome the complexity of 2D-evaluation and standardize the whole surgical reporting [7]. 


\section{References}

[1] Porpiglia F, Amparore D, Checcucci E, et al. Three-dimensional virtual imaging of renal tumours: a new tool to improve the accuracy of nephrometry scores. BJU international. 2019: 124:945-54

[2] Ficarra V, Novara G, Secco S, et al. Preoperative Aspects and Dimensions Used for an Anatomical (PADUA) Classification of Renal Tumours in Patients who are Candidates for NephronSparing Surgery. European urology. 2009 2009/11/01/: 56:786-93

[3] Kutikov A, Uzzo RG. The R.E.N.A.L. nephrometry score: a comprehensive standardized system for quantitating renal tumor size, location and depth. The Journal of urology. 2009: 182:84453

[4] Klatte T, Ficarra V, Gratzke C, et al. A Literature Review of Renal Surgical Anatomy and Surgical Strategies for Partial Nephrectomy. European urology. 2015: 68:980-92

[5] Schiavina R, Novara G, Borghesi $M$, et al. PADUA and R.E.N.A.L. nephrometry scores correlate with perioperative outcomes of robot-assisted partial nephrectomy: analysis of the Vattikuti Global Quality Initiative in Robotic Urologic Surgery (GQI-RUS) database. BJU international. 2017: 119:456-63

[6] Veccia A, Antonelli A, Uzzo RG, et al. Predictive Value of Nephrometry Scores in Nephronsparing Surgery: A Systematic Review and Meta-analysis. European urology focus. 2019:S24054569(19)30345-1

[7] Abaza R, Gerhard RS, Martinez O. Robotic Radical Nephrectomy for Massive Renal Tumors. Journal of laparoendoscopic \& advanced surgical techniques Part A. 2019:10.1089/lap.2019.0630

Figure 1

A) The picture shows anatomical structures 3D assessed (mass, renal shape, vasculature, collecting system [CS], ureter, and possible presence of renal cysts).

During arterial phase, vascularization results in the reconstruction of at least third or fourth order branches.

Depiction of two cases:

- Patient with a left kidney tumor, with (A-1) or without (A-2) captions from the DocDo tool; kidney removed (A-3); kidney and venal aspect removed (A-4); details of the inner relationship of the mass with the urinary colleting system (CS) $(A-5)$

- Patient with a right kidney tumor, medial rim, hilar proximity (B-1); kidney removed (B-2); kidney, venous aspect and CS removed, to focus on the arterious 
part and its proximity to the mass (B-3) ; posterior face of the same B patient, with the kidney in-site (B-4) to focus on the contact surface and protrusion of the mass (B-4); the same as B-4, without kidney and arterious aspect, to focus on the venous details and mass distance from the CS (B-5)

B) The Table shows the characteristics of the dataset, with nephrometric scoring; level of agreement between 3D and 2D nephrometric scoring is reported as Cohen's K. 

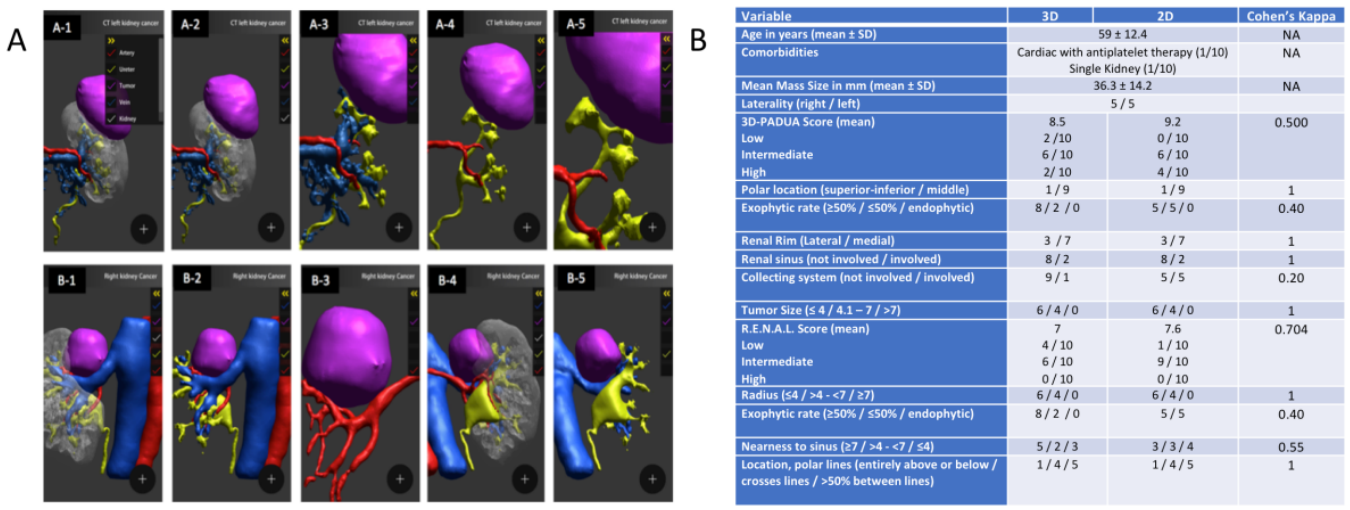

bju_15049_f1.tif 